\title{
A Network Pharmacology Approach for Exploring the Mechanisms of Panax notoginseng Saponins in Ischaemic Stroke
}

\author{
Cong Wang $\mathbb{D}^{D}$, Hao Chen $(\mathbb{D}$, Shi-tang Ma, Bin-bin Mao $(\mathbb{D}$, Yu Chen $(\mathbb{D})$, Hao-Nan Xu $(\mathbb{D}$, \\ and Hao Yu (D)
}

College of Life and Health Sciences, Anhui Science and Technology University, Fengyang 233100, China

Correspondence should be addressed to Hao Yu; yhz_1230@163.com

Received 30 January 2021; Revised 29 July 2021; Accepted 4 August 2021; Published 16 August 2021

Academic Editor: Francesca Mancianti

Copyright ( $\odot 2021$ Cong Wang et al. This is an open access article distributed under the Creative Commons Attribution License, which permits unrestricted use, distribution, and reproduction in any medium, provided the original work is properly cited.

\begin{abstract}
Background. Panax notoginseng saponins (PNS) have been deemed effective herb compounds for treating ischaemic stroke (IS) and improving the quality of life of IS patients. This study aimed to investigate the underlying mechanisms of PNS in the treatment of IS based on network pharmacology. Methods. PNS were identified from the Traditional Chinese Medicine System Pharmacology (TCMSP) database, and their possible targets were predicted using the PharmMapper database. IS-related targets were identified from the GeneCards database, OMIM database, and DisGeNET database. A herb-compound-target-disease network was constructed using Cytoscape, and protein-protein interaction (PPI) networks were established with STRING. GO enrichment and KEGG pathway analysis were performed using DAVID. The binding of the compounds and key targets was validated by molecular docking studies using AutoDock Vina. The neuroprotective effect of TFCJ was substantiated in terms of oxidative stress (superoxide dismutase, glutathione peroxidase, catalase, and malondialdehyde) and the levels of IGF1/PI3K/Akt pathway proteins. Results. A total of 375 PNS targets and 5111 IS-related targets were identified. Among these targets, 241 were common to PNS, and IS network analysis showed that MAPK1, AKT1, PIK3R1, SRC, MAPK8, EGFR, IGF1, HRAS, RHOA, and HSP90AA1 are key targets of PNS against IS. Furthermore, GO and KEGG enrichment analysis indicated that PNS probably exert therapeutic effects against IS by regulating many pathways, such as the Ras, oestrogen, FoxO, prolactin, Rap1, PI3K-Akt, insulin, PPAR, and thyroid hormone signalling pathways. Molecular docking studies further corroborated the experimental results. The network pharmacology results were further verified by molecular docking and in vivo experiments. Conclusions. The ameliorative effects of PNS against IS were predicted to be associated with the regulation of the IGF1-PI3K-Akt signalling pathway. Ginsenoside Re and ginsenoside Rb1 may play an important role in the treatment of IS.
\end{abstract}

\section{Introduction}

Stroke is a common disease worldwide, accounting for $11.59 \%$ of all deaths in 2019. Stroke, which accounts for more than $20 \%$ of China's total deaths, is the most common cause of death in China [1]. The main categories of stroke are ischaemic stroke (IS), cerebral haemorrhage, and subarachnoid haemorrhage [2]. In China, IS accounts for more than $80 \%$ of cases of stroke [1]. IS mainly occurs due to the obstruction of the middle cerebral artery, causing regional ischaemia and hypoxia in brain tissue, leading to DNA damage and cell apoptosis [3]. At present, there are no specific drugs for the clinical treatment of IS. Most of the drugs used for treatment are drugs that protect against free radical damage, thrombolytic drugs, antiplatelet aggregation drugs, anti-inflammatory drugs, and anticoagulants [4-6]. These drugs have serious side effects and a single mechanism of action, limiting their clinical use to a certain extent [7-10].

Traditional Chinese medicine compounds, which act through multiple components, pathways, and targets, have shown good clinical effects and thus are increasingly used to treat IS. This study provides new strategies for the treatment of IS in China. Panax notoginseng, as a traditional Chinese medicine, removes blood stasis, stops bleeding, promotes blood circulation, and relieves pain. The main active ingredients of Panax notoginseng are Panax notoginseng saponins 
(PNS), including notoginsenoside R1, ginsenoside $\mathrm{Rg} 1$, ginsenoside $\mathrm{Re}$, ginsenoside $\mathrm{Rbl}$, and ginsenoside $\mathrm{Rd}-\mathrm{qt}$ $[11,12]$. Studies have shown that PNS alleviate energy metabolism disturbances, balance ion metabolism, reduce the production of free radicals, and accelerate free radical scavenging. PNS exert antiapoptosis effects by maintaining mitochondrial homeostasis, inhibiting inflammation, protecting the blood-brain barrier (BBB), and increasing the blood supply to brain tissue [13]. However, the mechanism underlying the effect of PNS against IS is not completely clear.

Network pharmacology is a method of predicting drug targets and pharmacological mechanisms [14]. By predicting the complicated "drug-target-disease" relationship, it can assist the clinical evaluation of drug safety and effectiveness, reduce the cost of preclinical drug screening, and promote the development of new drugs. Additionally, multicomponent, multitarget, and regulatory networks based on network pharmacology can reveal the clinically complex mechanisms of action of drugs and are especially suitable for studying the mechanisms of Chinese herbal compounds and their complex components from the "holistic view" $[15,16]$. Previously, many scholars have demonstrated the predictive value of network pharmacology in in vivo and vitro experiments. Niu et al. [17] predicted that the ability of $A n$ gelica to treat acute myocardial infarction (AMI) might be related to the AMPK signalling pathway. This prediction was verified by in vitro and in vivo experiments. In vivo and in vitro verification experiments have indicated that Taohe Chengqi decoction may regulate the $\mathrm{PI} 3 \mathrm{~K} / \mathrm{AKT} / \mathrm{mTOR}$ and HIF-1 $\alpha$ /VEGF signalling pathways, consistent with the predicted results of network pharmacology [18]. Fu et al. [19] predicted that IGF1R is the main target of baicalin against epilepsy and verified through in vivo and in vitro experiments that baicalin inhibits IGF1R, thereby alleviating the symptoms of epilepsy. Dong et al. [20] predicted that VEGFA and BCL2A1 are the key targets of Panax notoginseng against cardiovascular diseases and verified this prediction by in vitro experiments. Zhang et al. [21] predicted that the PPAR signalling pathway is the key target pathway of Shen-Hong-Tong-Luo (SHTL) against atherosclerosis (AS) and verified that SHTL activates the PPAR pathway and reduces the inflammatory response and lipid accumulation in macrophages, thus reducing the symptoms of AS. Wen et al. [22] predicted that Chaiqin Chengqi decoction (CQCQD) may inhibit the TLR4/NLRP3 pathway, relieve inflammation, and reduce damage in acute pancreatitis (AP). The prediction results were verified by in vivo and in vitro experiments.

We explored the mechanism of PNS against IS through network pharmacology and provided a theoretical basis for the experimental research on the effect of PNS against IS and the efficacy of PNS in the clinical treatment of IS. The research process is shown in Figure 1.

\section{Materials and Methods}

2.1. Prediction of the Targets of PNS. The main PNS were identified from the Traditional Chinese Medicine Systems Pharmacology (TCMSP) database and analysis platform [23] (http://tcmspw.com/tcmsp.php), Integrative PharmacologyBased Research Platform of Traditional Chinese Medicine [24] (TCMIP, http://www.tcmip.cn/), and Bioinformatics Analysis Tool for Molecular Mechanism of Traditional Chinese Medicine [25] (BATMAN- TCM, http://bionet. ncpsb.org/batman-tcm/). The gene targets of the active compounds were downloaded from the PharmMapper database [26] (http://www.lilab-ecust.cn/pharmmapper/).

2.2. Prediction of IS-Related Targets. IS-related targets were identified from the following three disease-related databases: the GeneCards database (https:/www.genecards.org/), OMIM database (https://www.omim.org/), and DisGeNET database [27] (https://www.disgenet.org/). We used the keywords "brain infarction," "cerebral ischaemia," "cerebral infarction," and "stroke" to search these databases for ISrelated genes, merged the data from the databases, and deleted the duplicate data.

\subsection{Construction of the Protein-Protein Interaction (PPI)} Network. The PNS targets and IS-related targets that intersected were identified as common targets. The common PNS and IS-related targets were uploaded to the STRING 11.0 database (https://string-db.org/) [28], the type was set to "Homo sapiens," the confidence level was set to $>0.700$, the "hide disconnected nodes in the network" setting was simplified, the protein interaction information was obtained, the file was saved in the TSV format, and the data for node1, node2, and the combined score were imported into Cytoscape 3.7.2 [29] to establish a PPI network.

2.4. Establishment of an "Herb-Compound-Target-Disease" Network. The active compounds, common targets, PNS, and IS were arranged in a relationship pair file and then imported into Cytoscape 3.7.2 to construct a visual "herb-compoundtarget-disease" network.

2.5. Gene Set Enrichment Analysis. To further determine the mechanism of PNS against IS, the common PNS and IS targets were uploaded to the DAVID 6.8 database [30] (https://david.ncifcrf.gov/), the select identifier was limited to "OFFICIAL GENE SYMBOL," the list type was limited to "Gene List," and the species was limited to "Homo sapiens." Gene Ontology (GO) and KEGG pathway enrichment analysis of the common targets were performed with this database.

2.6. Molecular Docking Studies. Preprocessing structures were generated using AutoDock 4.2 (http://autodock. scripps.edu/). Docking simulations were performed using AutoDock Vina [31]. The crystal structures of the target proteins were downloaded from the Protein Data Bank (http://www.rcsb.org) [32]. The 2D structures of the natural ligands of the PNS compounds were downloaded from PubChem (https://pubchem.ncbi.nlm.nih.gov/). 


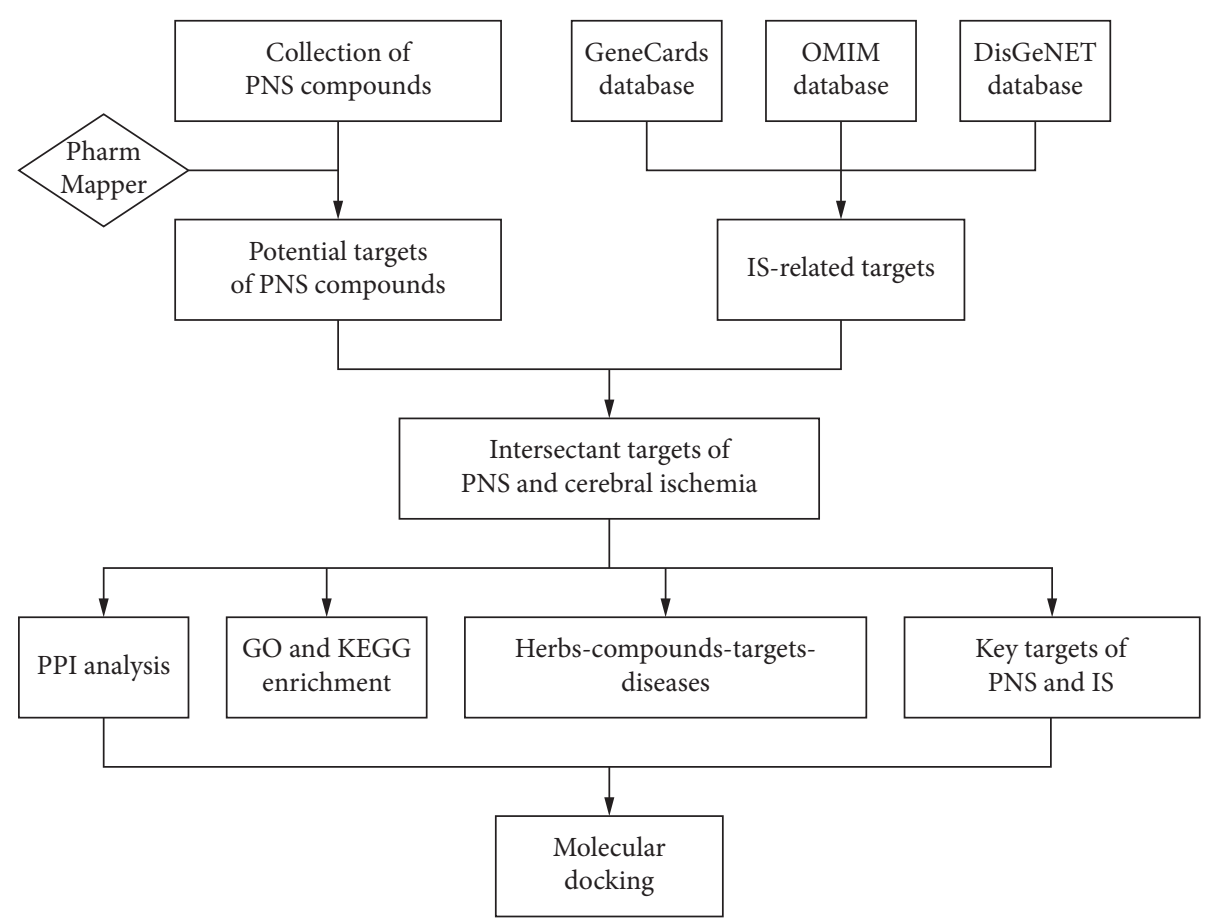

FIgURE 1: Flowchart of designed analysis in PNS against IS.

2.7. Middle Cerebral Artery Occlusion (MCAO) Establishment. Adult male Sprague-Dawley rats $(260 \pm 20 \mathrm{~g}$ bodyweight) were purchased from Huaxing Experimental Animal Farm in Huiji District, Zhengzhou City (Zhengzhou, Henan, China). All the animals were housed in an environment with a temperature of $23 \pm 2^{\circ} \mathrm{C}$, relative humidity of $55 \pm 5 \%$, and a light/dark cycle of $12 / 12 \mathrm{hr}$ with free access to food and water. In addition, all animal studies (including the mice euthanasia procedure) were performed in compliance with the regulations and guidelines of Anhui Science and Technology University Institutional Animal Care and conducted according to the AAALAC and the IACUC guidelines. Rats were randomly divided into six groups as follows: (1) Sham group received $10 \mathrm{~mL} / \mathrm{kg} 0.5 \%$ CMC daily, and the procedure was the same as that of MCAO except for the insertion of a filament; (2) MCAO group received $10 \mathrm{~mL} / \mathrm{kg}$ 0.5\% CMC solution daily before MCAO surgery. (3) PNS-L group received $40 \mathrm{mg} / \mathrm{kg}$ PNS (5 mg/pill, KPC Xuesaitong Pharmaceutical Co., Ltd.) daily before MCAO surgery. (4) PNS-H group received $80 \mathrm{mg} / \mathrm{kg}$ PNS daily before MCAO surgery; and (5) nimodipine group received $14.4 \mathrm{mg} / \mathrm{kg}$ nimodipine $(20 \mathrm{mg} /$ pill, Yabao Phamarceutical Group Co., Ltd.) daily before MCAO surgery. All the above rates were given intragastric administration once a day for 7 days.

Before the operation, the rats were fasted for $12 \mathrm{~h}$ to reduce the mortality during and after the operation and anesthetized by intraperitoneal injection of $2 \%$ pentobarbital sodium ( $4 \mathrm{~mL} / \mathrm{kg})$. According to the previous literature, we slowly inserted the threaded plug from the left external carotid artery (ECA) through the common carotid artery (CCA) into the internal carotid artery (ICA) until the middle cerebral artery (MCA) was blocked. After ischaemia $2 \mathrm{~h}$, the blood supply was restored, and reperfusion was achieved. The reperfusion process continued for $24 \mathrm{~h}$.

2.8. Oxidative Stress Evaluation. SOD, GSH-Px, CAT activities, and the content of MDA in the brain homogenate were determined according to manufacturer's instructions. All kits were purchased from Nanjing Jiancheng Bioengineering Institute.

2.9. Western Blot. The total proteins in the cerebral ischemia-reperfusion brain were were quantified with a bicinchoninic acid protein assay kit (P0010, Beyotime, Shanghai, China), loaded into $10 \%$ sodium dodecyl sulfatepolyacrylamide gel electrophoresis, and transferred to polyvinylidene fluoride (PVDF) membranes. After blocking with $5 \%$ fat-free milk or BSA for $1 \mathrm{~h}$, the PVDF membranes were incubated with primary antibodies at $4^{\circ} \mathrm{C}$ overnight (Table 1), following incubation with second antibody (66009-1-Ig, Proteintech, Wuhan, China) for 2 hours at room temperature. The protein bands were visualized using enhanced chemiluminescence (ECL), $\beta$-actin served as a loading control, and densitometric analysis was determined by Image J software.

2.10. Statistical Analysis. All data were expressed as mean \pm SD; SPSS 25.0 statistical software (IBM SPSS Statistics for Windows, IBM Corp.) was adopted for all statistical analyses. The multiple variables were performed by one-way analysis of variance (ANOVA), and Student"s $t$ tests were used for comparison of variable pairs. $P<0.05$ was considered as statistically significant. 
TABle 1: Primary antibodies for Western blot in this study.

\begin{tabular}{lcc}
\hline Primary antibodies & Company & Catalog \\
\hline Anti-AKT & Proteintech & 10176-2-AP \\
Anti-PI3K & Proteintech & $67071-1-$ Ig \\
Antiphospho-AKT (Ser473) & Proteintech & $66444-1-$ Ig \\
Antiphospho-PI3K & Proteintech & $66444-1-$ Ig \\
Anti-IGF & Proteintech & $28530-1-A P$ \\
Anti- $\beta$-actin & Proteintech & $66009-1-I g$ \\
\hline
\end{tabular}

\section{Results}

3.1. Predicted Targets of PNS. The details of the 5 compounds in PNS are described in Supplementary Table S1. The results retrieved from the PharmMapper databases were integrated to obtain the 300 targets with the highest matching degree with the five active PNS compounds. All targets were uploaded to the UniProt KB database and named uniformly. After deduplication, all information were merged, resulting in a database of 375 targets of PNS, including 196 common targets (Figure 2).

3.2. Screening of IS-Related Genes. Targets related to IS were identified from the GeneCards, OMIM, and DisGeNET databases. The targets were renamed using the UniProt KB database. After deduplication, all information was merged, resulting in a database of 5111 IS-related targets, including 934 common targets (Figure 3 ).

3.3. Construction of the PPI Network. A total of 241 common targets of IS and PNS were identified with a Venn tool. The network constructed with the STRING 11.0 database ignored disconnected targets, resulting in a PPI network of only 224 targets (Figure 4).

In the PPI network, there were a total of 224 nodes and 1119 edges. The three main parameters "degree (DC)," "closeness," and "betweenness (BC)" were set as filters to select core targets and construct the main hub of the node representing the effect of PNS against IS. The first screening threshold $\mathrm{DC} \geq 6, \mathrm{BC} \geq 132.806$, closeness $\geq 0.199$, and 73 nodes and 488 edges were identified. The second threshold $\mathrm{DC} \geq 17, \mathrm{BC} \geq 634.078$, closeness $\geq 0.220$, and 23 nodes and 124 edges were identified (Figure 5).

Based on these 23 core targets, we further constructed a "compound-core target" network (Figure 6). Ginsenoside Re was related to 19 targets, ginsenoside Rg1 was related to 19 targets, ginsenoside $\mathrm{Rb} 1$ was related to 20 targets, ginsenoside Rd_qt was related to 21 targets, and notoginsenoside R1 was related to 19 targets. The colour of each node represents the DC.

The triangular nodes represent compounds, and the circular node represents core targets.

SRC, MAPK1, NOS2, NR3C1, ALB, HRAS, NOS3, CASP3, MAPK8, RHOA, FABP5, HSP90AA1, BCL2L1, PRKACA, PIK3R1, F2, AKT1, IGF1, EGFR, MMP9, AR, RXRA, and ESR1 were arranged according to the DC (Table 2). The top ten targets were MAPK1 (58), AKT1 (50),

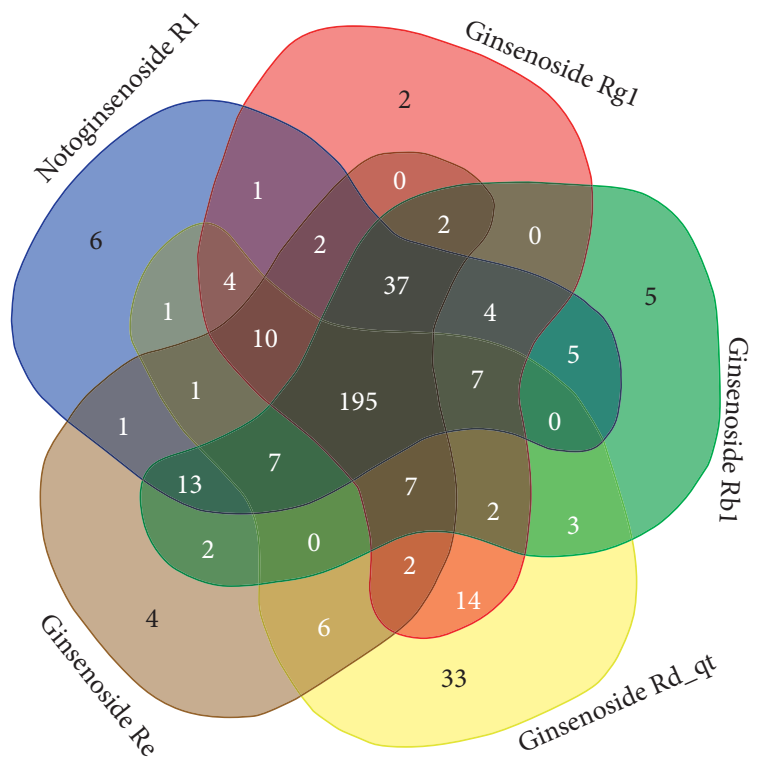

FIgURE 2: Venn diagram of the gene targets of PNS.

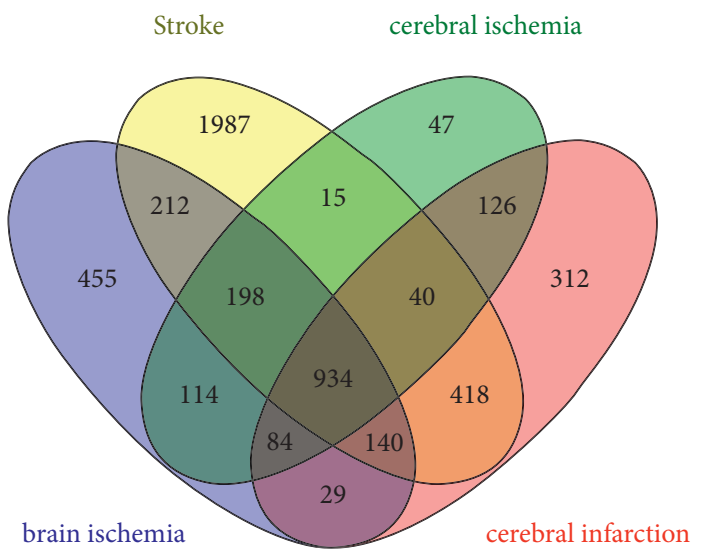

Figure 3: Venn diagram of gene targets related to IS.

PIK3R1 (47), SRC (45), MAPK8 (41), EGFR (41), IGF1 (38), HRAS (37), RHOA (35), and HSP90AA1 (34). These targets are the key targets of the PPI network.

\subsection{Establishment of an "Herb-Compound-Target-Disease"} Network. The herb-compound-target-disease network was composed of one herb, five compounds, 241 targets, and one disease. A diagram of this network is shown in Figure 7.

The circles represent PNS, the triangles represent PNS compounds, the squares represent the 241 targets, and the $\mathrm{V}$ represents IS.

3.5. Results of GO Enrichment and KEGG Pathway Analysis. We performed GO enrichment and KEGG pathway analysis of the common targets. For GO enrichment analysis (Supplementary Tables S2, S3, S4), we filtered the top five cellular components (CC), biological process (BP), and molecular function (MF) categories based on the following criteria: $p$ value $<0.01$ and FDR $<0.05$. The top 5 entries in the $\mathrm{BP}$ category were the steroid hormone-mediated 


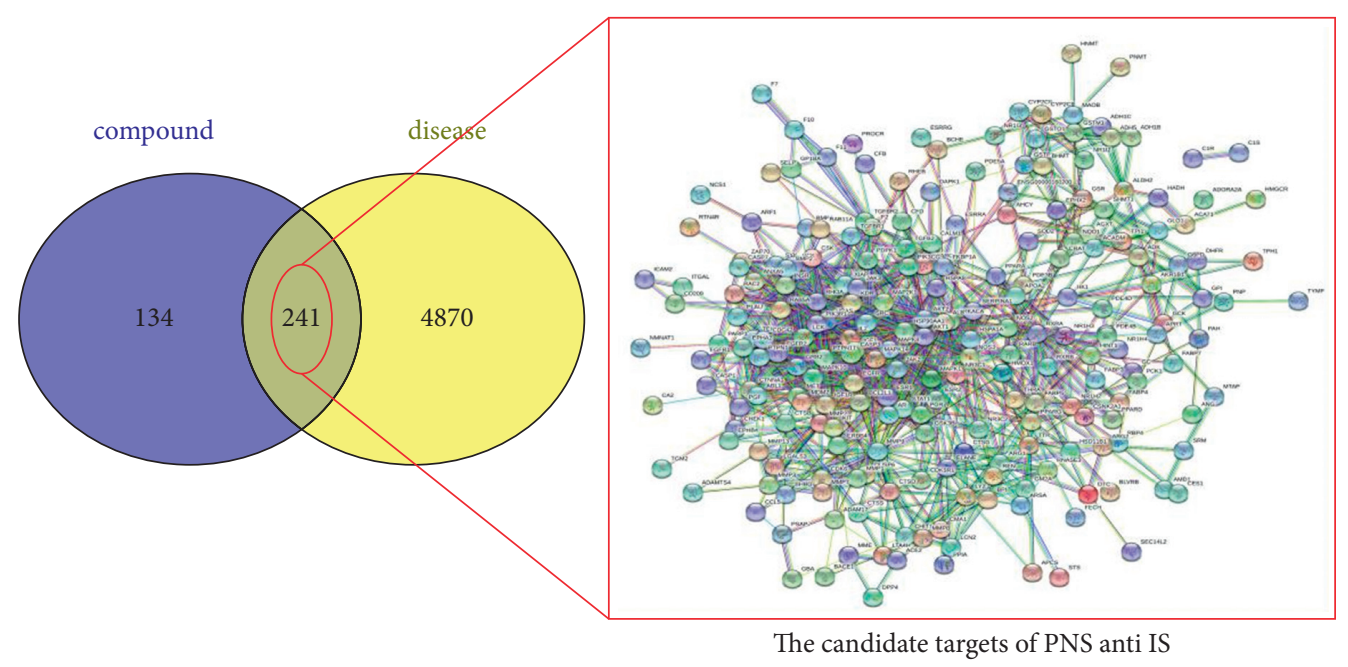

FIgURE 4: Venn diagram and PPI network of target genes of PNS and IS.

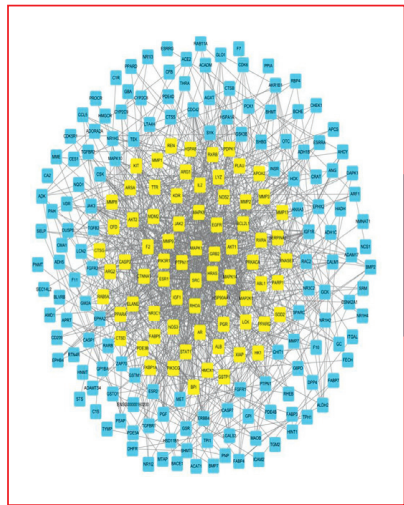

224 nodes and 1119 edges

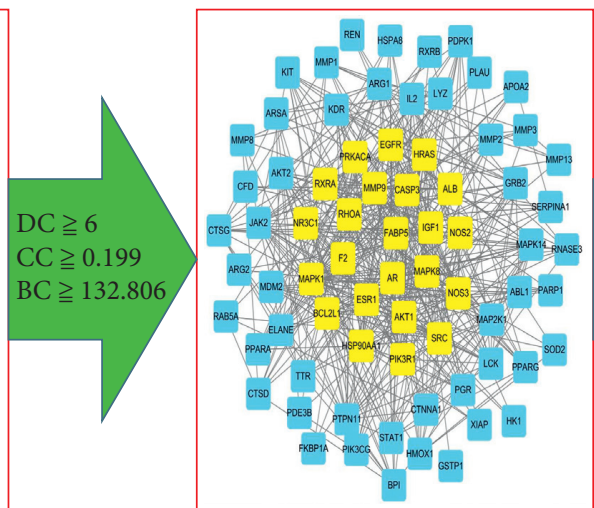

73 nodes and 488 edges

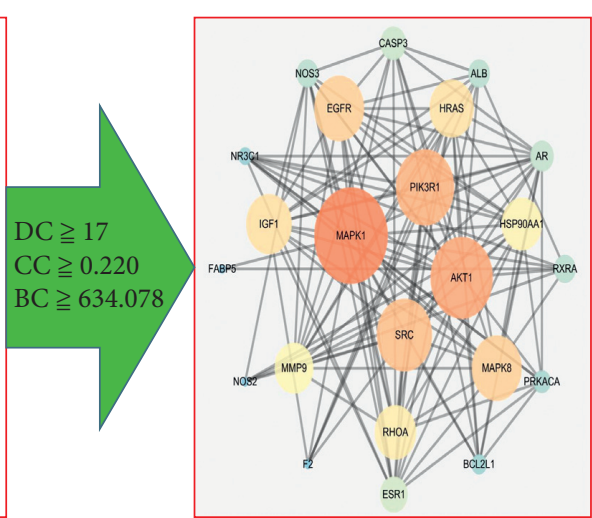

23 nodes and 124 edges

FIGURE 5: The screening process for the PPI network.

signalling pathway, transcription initiation from RNA polymerase II promoter, peptidyl-tyrosine autophosphorylation, protein autophosphorylation, and phosphatidylinositol-mediated signalling. The top 5 entries in the MF category were steroid hormone receptor activity, RNA polymerase II transcription factor activity, ligandactivated sequence-specific DNA binding, protein tyrosine kinase activity, and serine-type endopeptidase activity. The top 5 entries in the CC category were cytosol, extracellular exosome, extracellular space, extracellular region, and membrane raft. The results are shown in Figure 8.

To confirm the key pathways enriched in the targets of PNS against IS, we filtered the top 20 KEGG pathways (Supplementary Table S5). The entries were divided into five categories: biochemical substances involved in cancer (proteoglycans and central carbon metabolites), pathways in cancer, human diseases associated with cancer (pancreatic cancer, prostate cancer, colorectal cancer, chronic myeloid leukaemia, and nonsmall-cell lung cancer and glioma), signal transduction pathways (Ras, oestrogen, FoxO, prolactin, Rap1, PI3K-Akt, insulin, PPAR, and thyroid hormone signalling pathways); and types of cell junctions (adherens junctions). Specific information about the top 20 entries is shown in Figure 9.

3.6. Results of Molecular Docking Studies. We used binding energy to assess the effects of compound-target interactions. Docking of compounds and key targets was simulated, and the results are given in Table 3 and Figure 10. The binding energy of each stimulation was less than $-6 \mathrm{kcal} / \mathrm{mol}$, indicating molecular docking was effective. The highest docking scores are shown in Figure 11.

3.7. PNS Reduces Oxidative Stress in MCAO Rats. The activity of SOD, GSH-Px, and CAT in the brain tissue was higher in the MCAO group than in the sham group (Figures 12(a)$\left.12(c),{ }^{\#} p<0.01\right)$, and the concentration of MDA was lower in the MCAO group than in the sham group (Figure 12(d), \#\# $p<0.01)$.

The activity of SOD, GSH-Px, and CAT in the brain tissue of the PNS group was much greater than in the MCAO group (Figures $12(\mathrm{a})-12(\mathrm{c}),{ }^{* *} p<0.01$ ). The concentration of MDA in the brain tissue of the PNS group was 


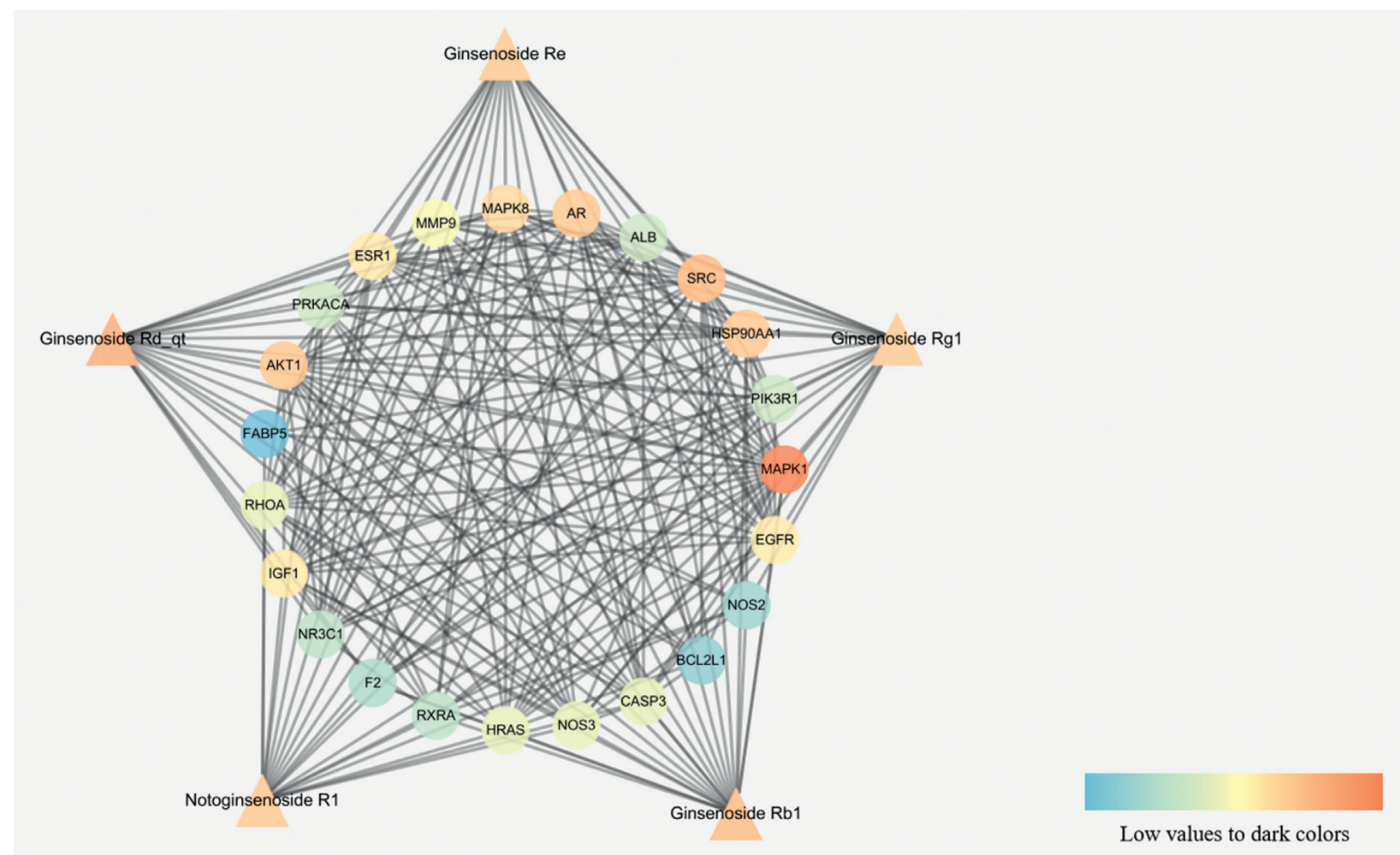

Figure 6: The compound-core target connection network.

TABLe 2: Degree information for core targets.

\begin{tabular}{|c|c|c|c|}
\hline Gene names & Protein names & Degree & Length \\
\hline SRC & Protooncogene tyrosine-protein kinase Src & 58 & 360 \\
\hline MAPK1 & Mitogen-activated protein kinase 1 & 50 & 480 \\
\hline NOS2 & Nitric oxide synthase type II & 47 & 724 \\
\hline NR3C1 & Nuclear receptor subfamily 3 group C member 1 & 45 & 536 \\
\hline ALB & Albumin & 41 & 427 \\
\hline HRAS & GTPase HRas & 41 & 1210 \\
\hline NOS3 & Nitric oxide synthase type III & 38 & 195 \\
\hline CASP3 & Caspase-3 & 37 & 189 \\
\hline MAPK8 & Mitogen-activated protein kinase 8 & 35 & 193 \\
\hline RHOA & Transforming protein RhoA & 34 & 732 \\
\hline FABP5 & Fatty acid-binding protein 5 & 33 & 707 \\
\hline HSP90AA1 & Heat shock protein HSP 90 -alpha & 27 & 595 \\
\hline BCL2L1 & Bcl-2-like protein 1 & 26 & 277 \\
\hline PRKACA & Protein kinase $\mathrm{C}$ alpha type & 25 & 920 \\
\hline PIK3R1 & Phosphatidylinositol 3-kinase regulatory subunit alpha & 24 & 609 \\
\hline $\mathrm{F} 2$ & Coagulation factor II & 24 & 1203 \\
\hline AKT1 & AKT serine/threonine kinase 1 & 24 & 462 \\
\hline IGF1 & Insulin-like growth factor I & 22 & 672 \\
\hline EGFR & Epidermal growth factor receptor & 21 & 233 \\
\hline MMP9 & Matrix metalloproteinase- 9 & 20 & 777 \\
\hline $\mathrm{AR}$ & Androgen receptor & 17 & 1153 \\
\hline RXRA & Retinoic acid receptor RXR-alpha & 17 & 135 \\
\hline ESR1 & Estrogen receptor alpha & 17 & 622 \\
\hline
\end{tabular}

significantly lower compared to that in the MCAO group (Figure 12(d), ${ }^{* *} p<0.01$ ).

3.8. The Protein Expression of the IGF/PI3K/AKT Pathway Mediated by PNS. As the top 20 of the KEGG pathway, the PI3K-Akt signalling pathway played an important role, and
IGF was its upstream protein. Therefore, we focused on the regulation of PNS on the protein of IGF and its downstream PI3K-Akt signal pathway. The IGF1/ $\beta$-actin, p-Akt/ Akt, and p-mTOR/mTOR expression in the MCAO control rats were significantly lower than in the sham rats (Figure 13(b), ${ }^{\# \#} p<0.01$ ). The IGF1/ $\beta$-actin, p-Akt/Akt, 


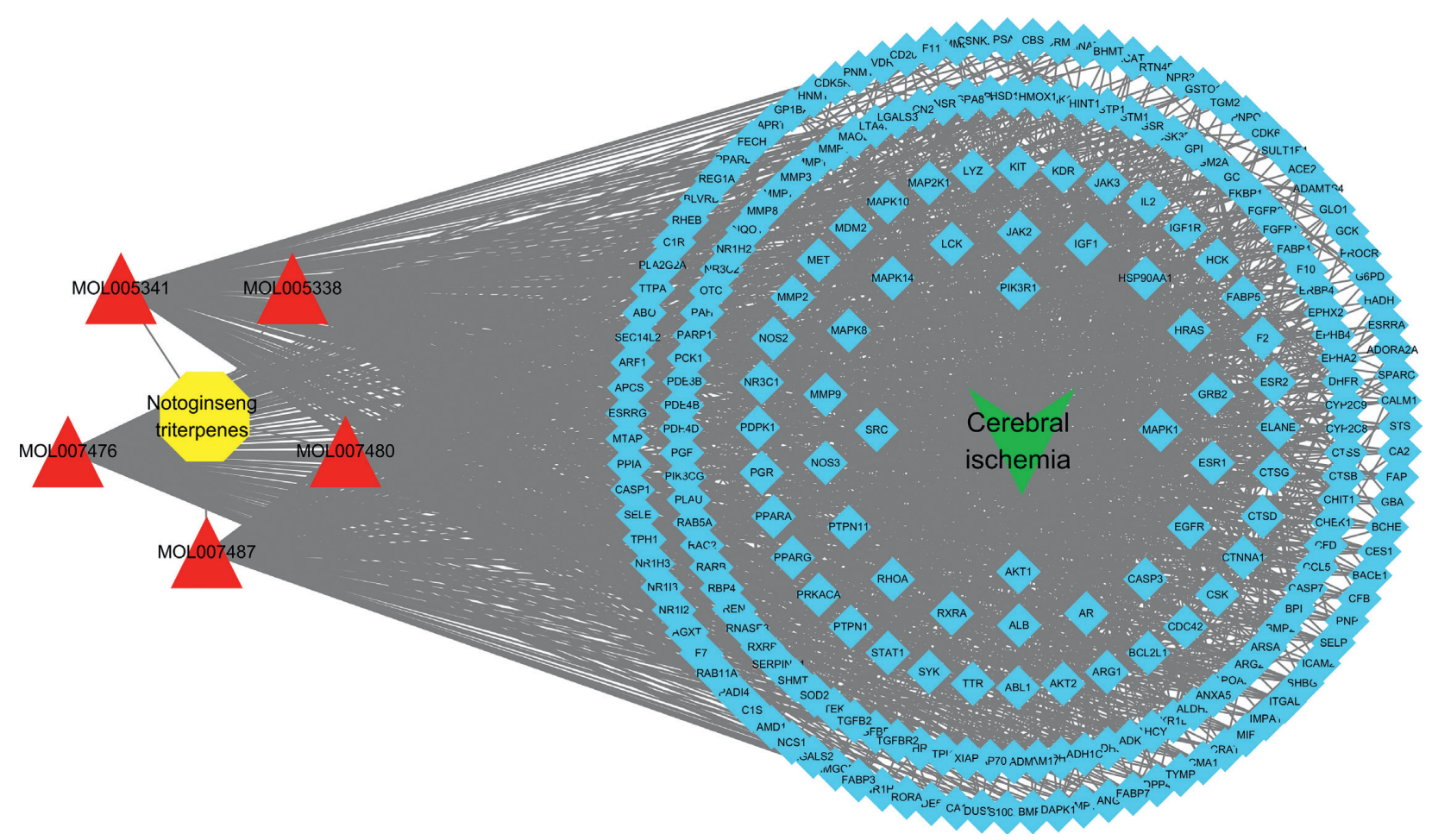

Figure 7: Herb-compound-target-disease network.

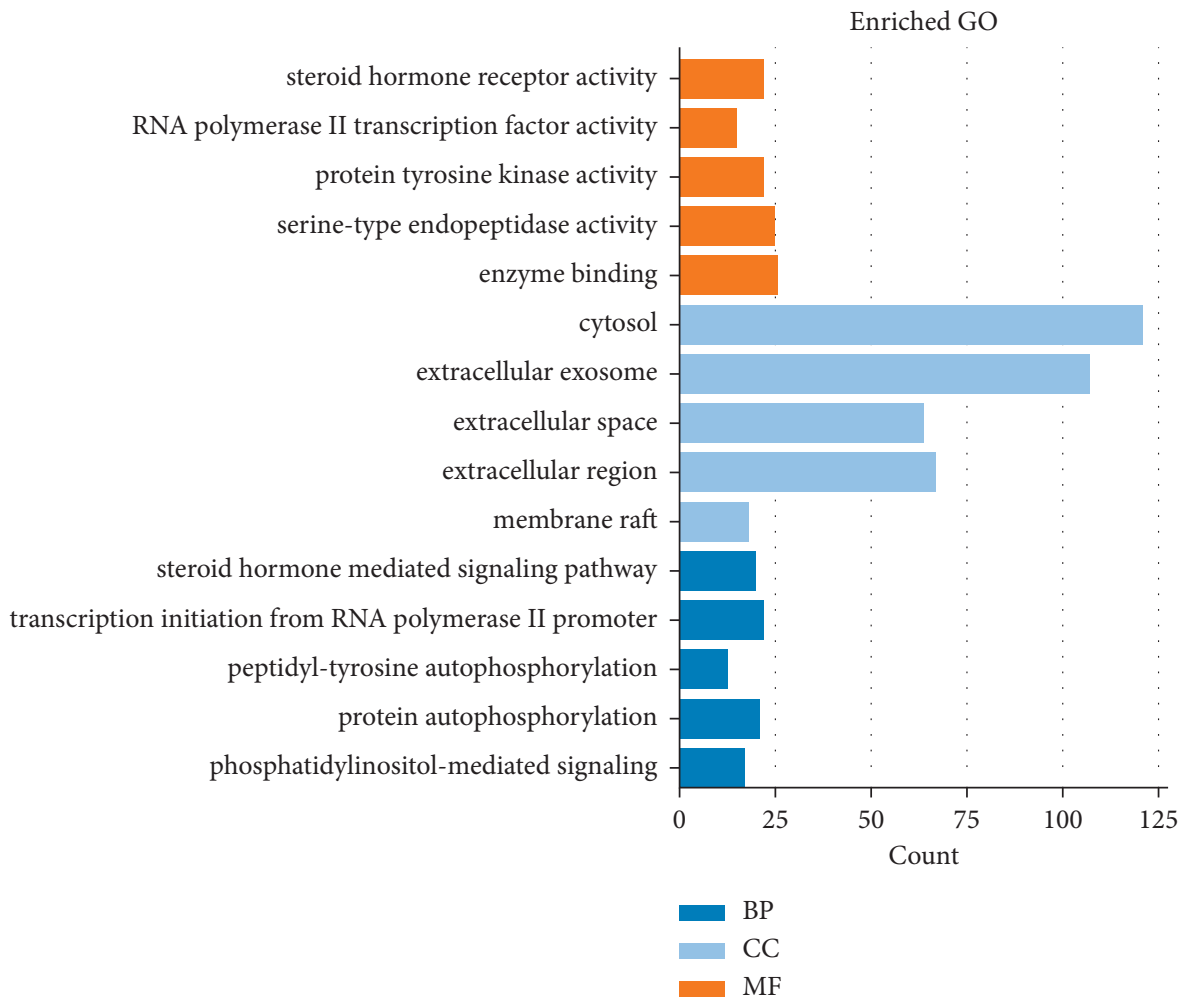

Figure 8: GO enrichment analysis of the 241 nodes (BP, MF, and CC categories). 


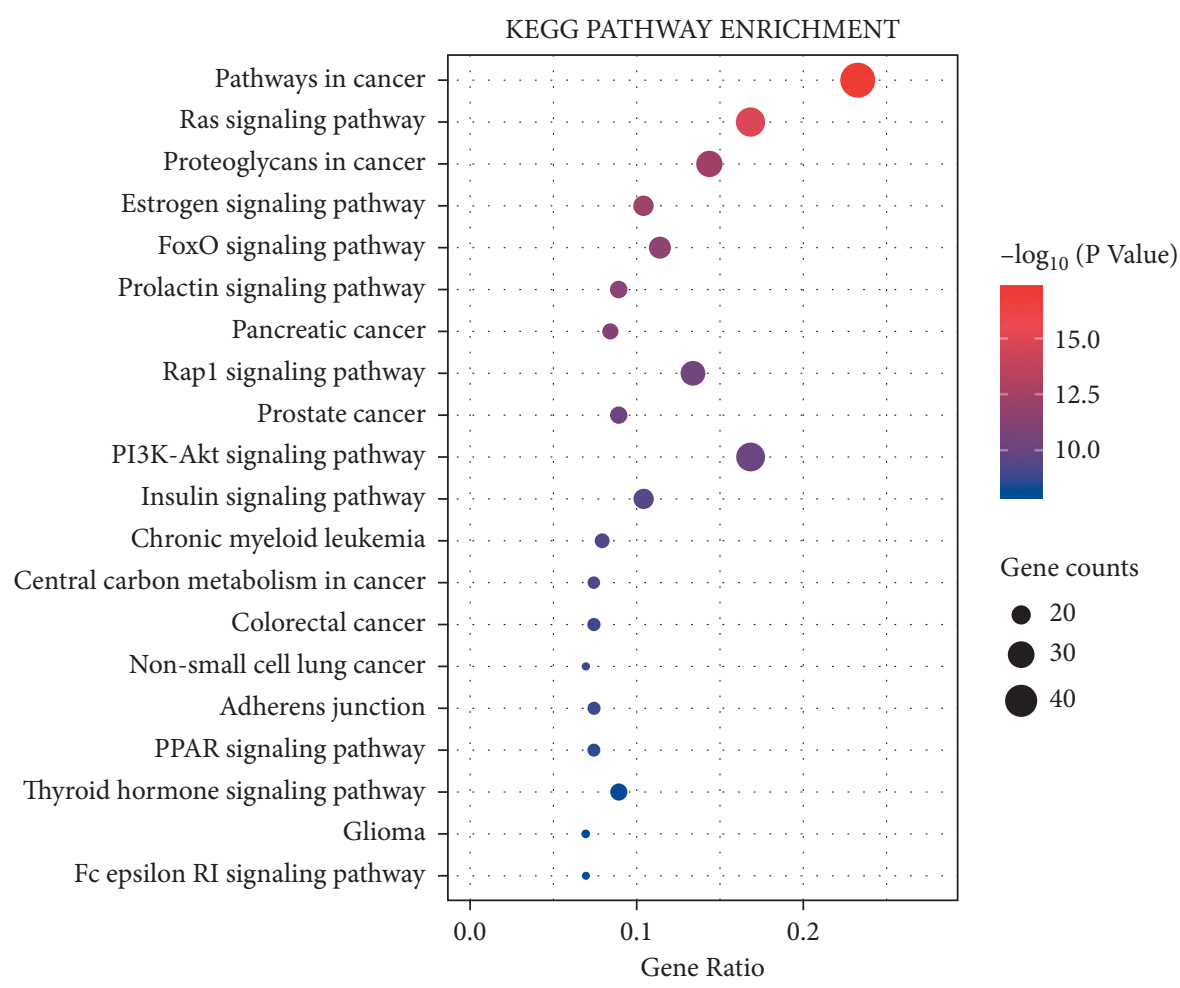

FIGURE 9: Top 20 enriched KEGG pathways.

TABLE 3: Docking information for 10 targets and their corresponding compounds.

\begin{tabular}{|c|c|c|c|c|c|c|c|c|c|c|c|}
\hline \multirow{2}{*}{ CAS } & \multirow{2}{*}{ Molecule name } & \multicolumn{10}{|c|}{ Docking score $(\mathrm{kcal} / \mathrm{mol})$} \\
\hline & & HRAS & SRC & MAPK1 & HSP90AA1 & MAPK8 & EGFR & RHOA & AKT1 & PIK3R1 & IGF1 \\
\hline $51542-56-4$ & Ginsenoside $\operatorname{Re}$ & -6.5 & -9.8 & -7.3 & -8.1 & -7.9 & -7.3 & -7.4 & -7.5 & -8.5 & -8.8 \\
\hline $22427-39-0$ & Ginsenoside Rg1 & -5.6 & -8 & -7.5 & -7.2 & -7.0 & -6.8 & -7.4 & -7.3 & -9.4 & -8.6 \\
\hline $41753-43-9$ & Ginsenoside Rb1 & -6.6 & -10 & -7.1 & -8.2 & -7.3 & -7.3 & -8 & -6.8 & -8.7 & -7.5 \\
\hline $62025-49-4$ & Ginsenoside Rd_qt & -6.4 & -8.4 & -7.6 & -6.9 & -7.1 & -7.3 & -7.8 & -6.5 & -7.4 & -7.8 \\
\hline $80418-24-2$ & Notoginsenoside R1 & -6.1 & -9.3 & -7.7 & -8.4 & -7.2 & -6.1 & -7.5 & -6.9 & -8.6 & -8.2 \\
\hline
\end{tabular}

and $\mathrm{p}-\mathrm{mTOR} / \mathrm{mTOR}$ expression in the PNS treatment rats were significantly higher than in the MCAO control rats (Figure 13(b), ${ }^{* *} p<0.01$ ). There was no significant change in total PI3K and Akt protein expression in each group $(p>0.05)$.

\section{Discussion}

IS is a clinically acute disease. Within the first few minutes to a few hours after intracerebral artery occlusion, the infarcted area containing ischaemic tissue gradually spreads outward. As time passes after reperfusion, the ability of brain tissue to recovery decreases gradually [33]. Arterial occlusion is thought to cause the apoptosis of 2 million neurons per minute, and the number of neurons that becomes apoptotic within 10 hours is equivalent to the number of neurons that becomes apoptotic over 36 years of normal ageing [34]. The current treatment plan addresses the changes observed in animal models of local cerebral ischaemia and reperfusion. The primary goal is to relieve arterial obstruction as soon as possible, achieve recanalization, restore cerebral blood flow,

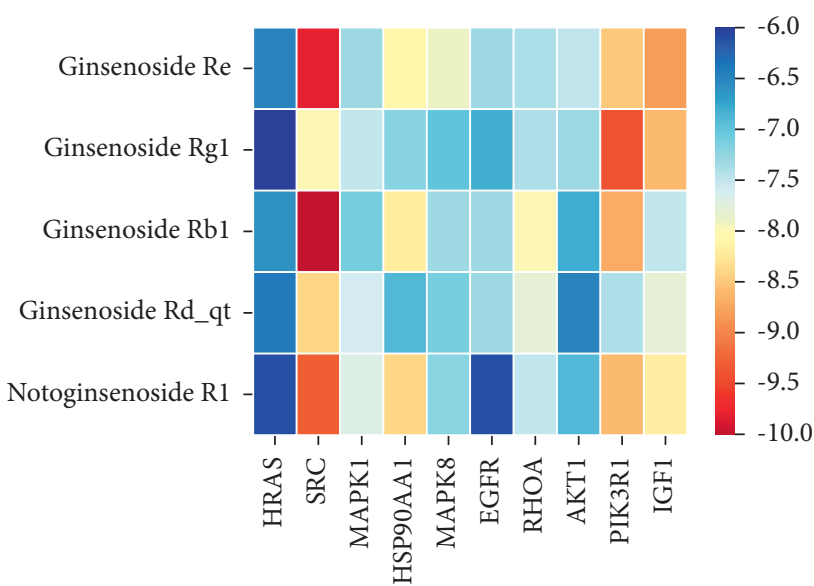

FIGURE 10: Docking information for 10 targets and their corresponding compounds.

and achieve reperfusion to reduce tissue damage and relieve symptoms [33]. Reteplase [8], a commonly used thrombolytic drug, has side effects, such as visceral bleeding. 


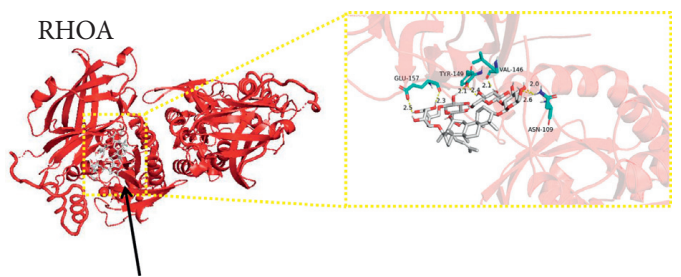

Ginsenoside Rb1

(a)

AKT1

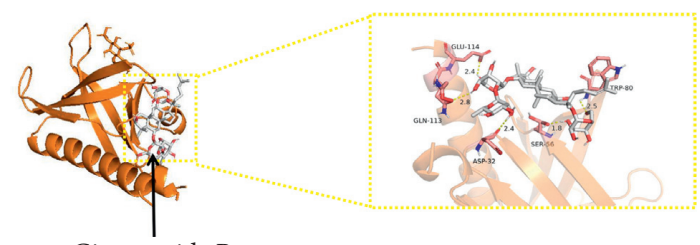

(c)

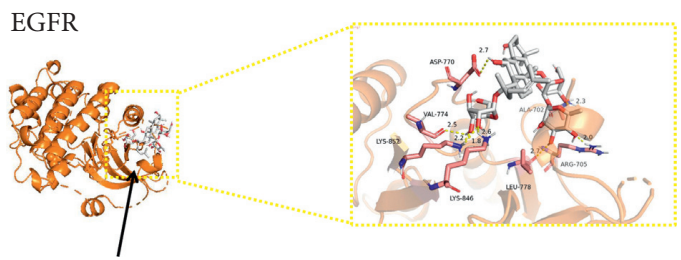

Ginsenoside Re

(e)

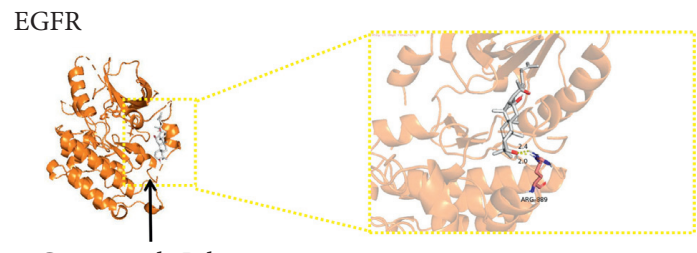

Ginsenoside Rd_qt

(g)

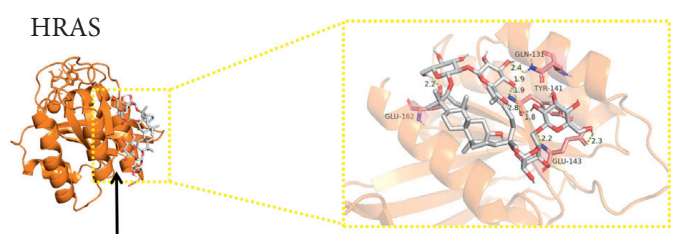

Ginsenoside Rb1

(i)

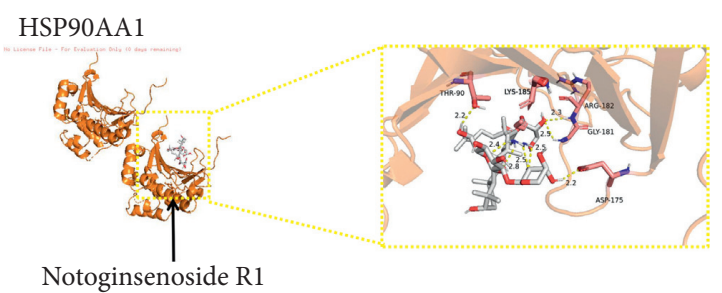

(k)

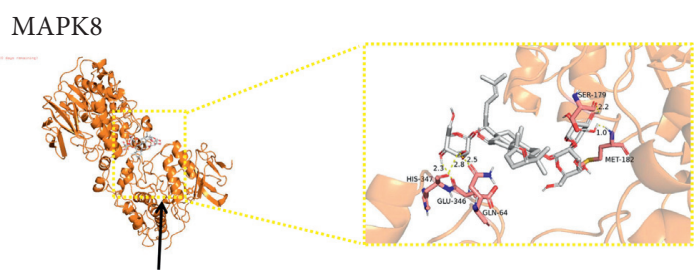

Ginsenoside $\mathrm{Rb}$

(b)

IGF1
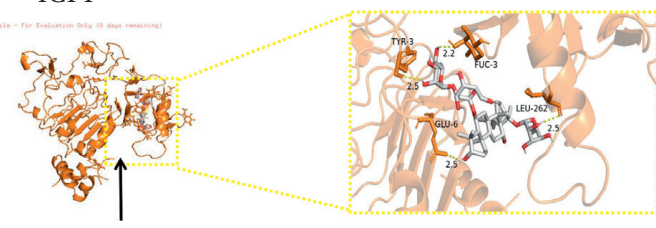

Ginsenoside Re

(d)

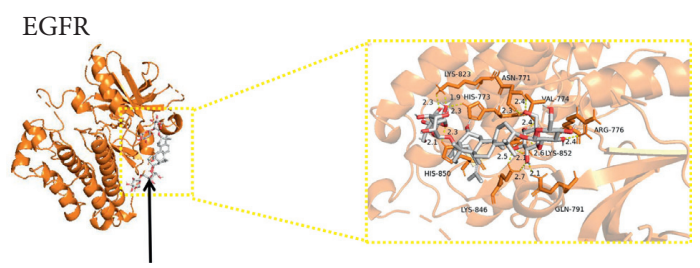

Ginsenoside Rb1

(f)

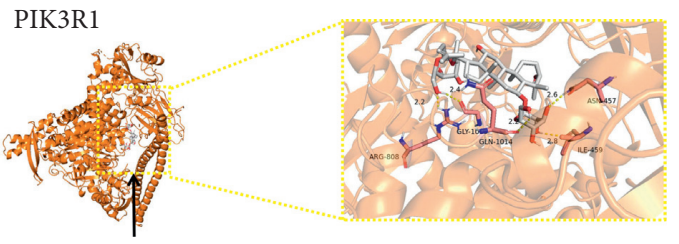

Ginsenoside Rg1

(h)

MAPK1

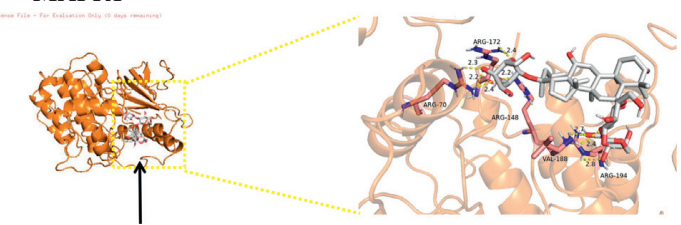

Notoginsenoside R1

(j)

SRC

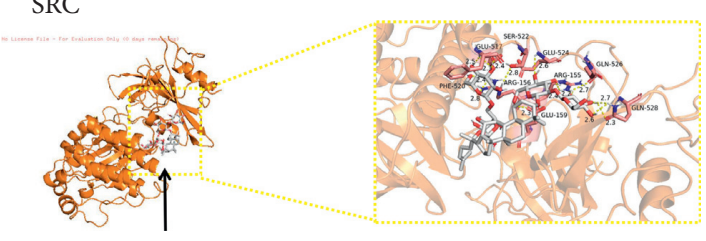

Ginsenoside Rb1

(1)

FIgURE 11: Detailed target compound interactions in the docking simulation. (a) RHOA protein ginsenoside Rb1; (b) MAPK8 protein ginsenoside Re; (c) AKT1 protein ginsenoside Re; (d) IGF1 protein ginsenoside Re; (e) EGFR protein ginsenoside Re; (f) EGFR protein ginsenoside Rb1; (g) EGFR protein ginsenoside Rd_qt; (h) PIK3R1 protein ginsenoside Rg1; (i) HRAS protein ginsenoside Rb1; (j) MAPK1 protein notoginsenoside R1; (k) HSP90AA1 protein notoginsenoside R1; (l) SRC protein ginsenoside Rb1. 


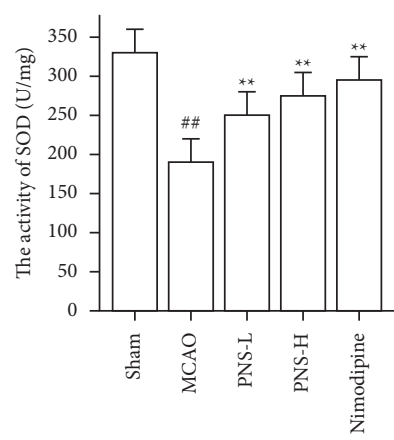

(a)

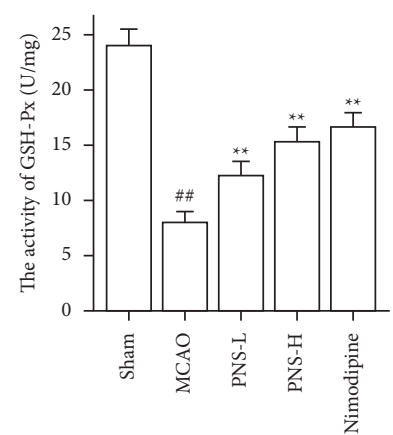

(b)

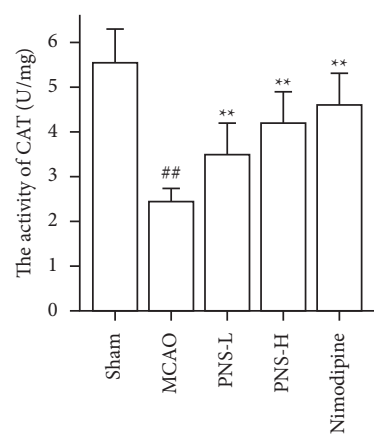

(c)

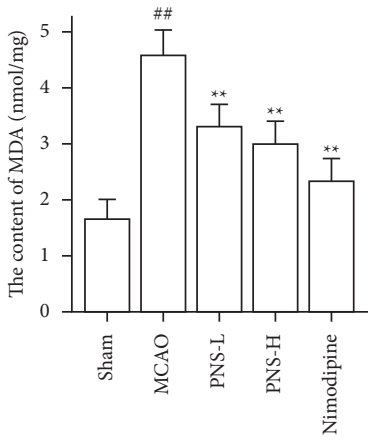

(d)

FIGURE 12: Effects of PNS on MCAO inducing oxidative stress. (a) SOD activity; (b) GSH-Px activity; (c) CAT activity; (d) MDA level. Results were showed as mean $\pm \mathrm{SD}\left(n=5 ; n\right.$, numbers of rats), ${ }^{\#} P<0.05,{ }^{\# \#} p<0.01$ vs. the sham group. ${ }^{*} P<0.05,{ }^{* *} p<0.01$ vs. the MCAO group.

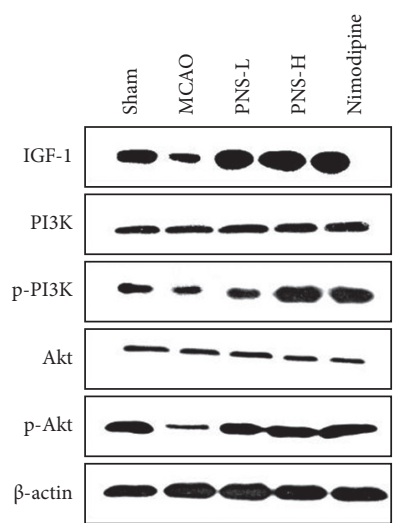

(a)

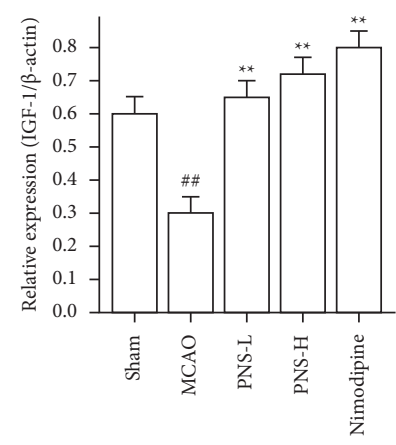

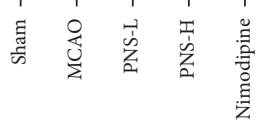

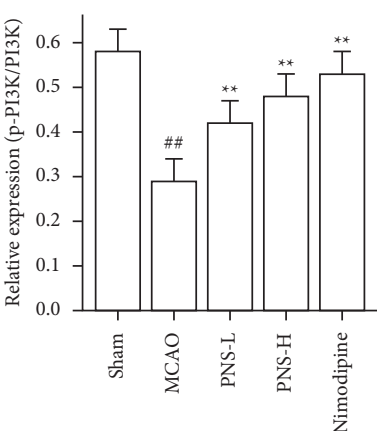

(b)

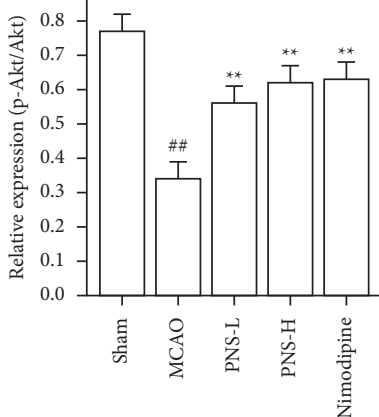

Figure 13: The effects of PNS on the IGF/PI3K/Akt pathway. (a) The IGF1, Akt, mTOR, p-Akt, and p-mTOR expression were measured using Western blotting. (b) The data of IGF1/ $\beta$-actin, p-Akt/Akt, and p-mTOR/mTOR expression. Results were shown as mean $\pm \mathrm{SD}(n=5$, $n$ : numbers of rats), ${ }^{\#} P<0.05,{ }^{\# \#} p<0.01$ vs. the sham group. ${ }^{*} P<0.05,{ }^{* *} P<0.01$ vs. the MCAO group.

Dexamethasone $[9,10]$, a commonly used anti-inflammatory drug, has side effects, such as inhibition of the body's immune function and induction of glucose metabolism disorders and osteoporosis. Studies have reported that PNS or their active compounds can reduce ROS production, relieve inflammation, inhibit cell apoptosis, and protect neurons. PNS have not been reported to have any side effects. Notoginsenoside R1 [35] reduces cerebral hypoxicischaemic damage in neonatal rats. Ginsenoside Rg1 [36] has a neuroprotective effect against cerebral ischemia-reperfusion injury. In particular, pretreatment with ginsenoside Rdqt alone alleviates early MCAO-induced damage in rats. Moreover, administration of ginsenoside $\mathrm{Rd}$-qt to rats within 4 hours after MCAO injury has an apparent neuroprotective effect. The therapeutic window of thrombolytic drugs is $6-12$ hours after onset $[37,38]$. In comparison, ginsenoside Rd-qt can improve the prognosis of patients 72 hours after IS onset [39].

Most Chinese medicines regulate the expression of pathways by acting on multiple genes or proteins and usually do not have serious side effects. For this reason, it is impossible to confirm how traditional Chinese medicines affect protein expression in organisms. However, network pharmacology can determine the potential molecular mechanism of drugs with multiple targets. Network pharmacology mainly involves prediction of compound and disease targets, construction of networks, PPI analysis, GO and KEGG enrichment analysis, and molecular docking studies and was used here to evaluate the mechanism underlying the therapeutic effect of PNS in IS.

In this study, it was confirmed that PNS and IS have 224 common gene targets; a PPI network was constructed; DC, $\mathrm{BC}$, and closeness were determined for two screenings; and 23 targets were identified. The targets with the top ten DCs were MAPK1, AKT1, PIK3R1, SRC, MAPK8, EGFR, IGF1, HRAS, RHOA, and HSP90AA1, which are the key targets.

According to GO analysis, the targets of PNS against IS regulate $\mathrm{BP}$ terms, affecting $\mathrm{CC}$ and $\mathrm{MF}$ terms. The results showed that PNS mainly regulate the steroid hormonemediated signalling pathway, RNA polymerase II promoter transcription, autophosphorylation of peptidyl-tyrosine, autophosphorylation of proteins, and phosphatidylinositolmediated signalling. It was also shown that PNS affects specific CC and MF terms, including cytosol, extracellular 
region, steroid hormone receptor activity, RNA polymerase II transcription factor activity, protein tyrosine kinase activity, and serine endopeptidase activity.

KEGG enrichment analysis indicated that that the targets were mainly enriched in signalling pathways, cell connection types, cancer-related diseases, biochemical substances, and pathways. According to reports in the literature, inhibition of the expression of related proteins in the Ras-MEK-ERK pathway can inhibit vascular intimal hyperplasia after stroke and reduce neuronal damage [40-42]. Activation of the $\operatorname{PPAR} \gamma$-Nrf2-NF- $\kappa \mathrm{B}$ signalling pathway can reduce neuroinflammation after stroke [43]. Activation of the PI3K-AktFOXO pathway inhibits the transcription of FOXO-3a and promotes the proliferation of neuronal stem cells [44]. The prolactin, oestrogen, and Rap1 signalling pathways can also activate the PI3K-Akt signalling pathway and inhibit cell apoptosis [45-47]. Therefore, it can be predicted that PNS mainly exerts its effect against IS through PI3K- and Aktrelated proteins. Akt usually acts as a critical regulator of cell growth, apoptosis, and proliferation, while PI3K mainly activates Akt. The PI3K-Akt signalling pathway has a neuroprotective effect in MCAO rats through inhibition of cell apoptosis [48, 49]. By activating the PI3K-Akt signalling pathway, Danhong injection increases the expression of antiapoptotic factors, inhibits the expression of apoptotic factors, and exerts a neuroprotective effect in MCAO rats [50]. Electroacupuncture reduces the cerebral infarct volume and the activation of cleaved caspase- 3 in MCAO rats by activating the PI3K-Akt signalling pathway [51]. Ligustilideinduced activation of the PI3K-Akt signalling pathway inhibits hippocampal neuron apoptosis in two models of oxygen-glucose deprivation/reperfusion (OGD/R) and in MCAO rats [52]. SOX5 promotes the expression of VEGF, which activates the PI3K-Akt signalling pathway and protects nerves in MCAO rats [53]. PNS activate the Nrf2 antioxidant signal through the PI3K/Akt signalling pathway to prevent $\mathrm{BBB}$ damage induced by $\mathrm{OGD} / \mathrm{R}$ [54]. Maintaining $\mathrm{BBB}$ integrity is a critical strategy for the prevention of IS [55].

Notoginsenoside $\mathrm{Rb} 1$ regulates the Akt-mTOR-PTEN signalling pathway and can alleviate motor and cognitive impairment induced by MCAO in rats [56]. PNS may improve the survival of endothelial cells in MCAO mice through the PI3K-Akt signalling pathway and reduce the cytotoxicity of leukocytes [57].

The results of the network pharmacology study were also confirmed by molecular docking studies. Generally, a binding free energy lower than $-5.0 \mathrm{kcal} / \mathrm{mol}$ indicates that the docking molecule has excellent binding activity with the target; a binding energy lower than $-7.0 \mathrm{kcal} / \mathrm{mol}$ indicates that the binding force is strong and that there is a significant interaction. The binding energies of the compounds and core targets were less than $-6.0 \mathrm{kcal} / \mathrm{mol}$, indicating good docking and high binding activity; in particular, the binding energy of SRC and ginsenoside Rb1 was $-10.0 \mathrm{kcal} / \mathrm{mol}$. Ginsenoside $\mathrm{Rb} 1$ protects the endothelial barrier function through the suppression of Src activation [58]. From the perspective of molecular interactions, the results also verified the network pharmacology data.
IGF1, acts as a ligand for IGF1R, activated the PI3K-AKT [59] and Ras-MAPK [6] pathways. PIK3R1 and AKT1 were the key proteins of the PI3K-AKT [59] pathways which regulate apoptosis and oxidative stress. EGFR activated epidermal growth factor, initiated a cascade of downstream signalling events leading to activation of the RAS-RAFMEK-ERK [60] and PI3K-AKT [61] pathways, and improved the condition of cerebral infarction. MAPK1 and MAPK8 [62] which act as the essential components of the MAP kinase signal transduction pathway involved in cell proliferation, differentiation, migration, and programmed cell death, that was a major downstream signalling pathway of the epidermal growth factor. HSP90AA1 [63] is a molecular chaperone that is involved for instance in the signal transduction and interaction with cochaperone proteins. HRAS [64] was involved in the activation of Ras protein signal transduction and decreased cell apoptosis. Most of the key gene targets are related to the PI3K-AKT pathway. Therefore, the PI3K/Akt pathway plays an important role in PNS anti-IS.

In our study, we found that PNS upregulated the activities of SOD, GSH-Px, and CAT after IS $24 \mathrm{~h}$, whereas PNS downregulated the MDA level, and PNS increased the ratio of IGF1/ $\beta$-actin, p-Akt/Akt, and p-mTOR/mTOR after IS $24 \mathrm{~h}$. These results showed PNS anti-IS through the PI3KAKT pathway by MCAO rats.

\section{Conclusions}

In this study, the targets, mechanism of action, and associated signalling pathways of PNS in the treatment of IS were predicted through network pharmacology; the binding abilities of the active ingredients of PNS and their targets were verified; and a fundamental basis for elucidating the mechanism of action of PNS was provided. Ginsenoside Rb1 and ginsenoside Rd-qt were related to most core targets. The key targets in the PPI network of the effect of PNS against IS were MAPK1, AKT1, PIK3R1, SRC, MAPK8, EGFR, IGF1, HRAS, RHOA, and HSP90AA1. According to molecular docking analysis, ginsenoside Re and ginsenoside Rb1, the active components of PNS, bind well with the key targets SRC, MAPK1, AKT1, and PIK3R1. Ginsenoside Re and ginsenoside Rb1 may play an essential role in IS treatment. The IGF1/PI3K-Akt signalling pathway, as a strongly associated pathway verified by in vivo experiments, may be used as a critical pathway underlying the effect of PNS against IS. Network pharmacology helps to reveal the mechanisms through which Chinese herbs can treat diseases.

\section{Data Availability}

The data used to support the findings of this study are available from the corresponding author upon request.

\section{Conflicts of Interest}

The authors declare that they have no conflicts of interest. 


\section{Acknowledgments}

The authors sincerely thank Collaborative Innovation Project of Colleges and Universities in Anhui Province (GXXT-2019-043) for its support. The present study was supported by the Key Research and Development Plan Projects in Anhui Province (202004a07020031) and the Project of Natural Science Foundation of Anhui Province (2008085QH395).

\section{Supplementary Materials}

The supplementary materials are available online. Table S1: basic information of ingredients in PNS; Table S2: functions of potential target genes based on the GO molecular function; Table S3: functions of potential target genes based on the GO biological process; Table S4: functions of potential target genes based on the GO cellular component; Table S5: functions of potential target genes based on KEGG analysis. (Supplementary Materials)

\section{References}

[1] GBD 2019 Demographics Collaborators, "Global age-sexspecific fertility, mortality, healthy life expectancy (HALE), and population estimates in 204 countries and territories, 1950-2019: a comprehensive demographic analysis for the Global Burden of Disease Study 2019," Lancet, vol. 396, no. 10258, pp. 1160-1203, 2020.

[2] S. Garbuzova-Davis, E. Haller, N. Tajiri et al., "Blood-spinal cord barrier alterations in subacute and chronic stages of a rat model of focal cerebral ischemia," Journal of Neuropathology \& Experimental Neurology, vol. 75, no. 7, pp. 673-688, 2016.

[3] P. Li, R. A. Stetler, R. K. Leak et al., "Oxidative stress and DNA damage after cerebral ischemia: potential therapeutic targets to repair the genome and improve stroke recovery," Neuropharmacology, vol. 134, pp. 208-217, 2018.

[4] T. Kanazawa, T. Kurano, H. Ibaraki, Y Takashima, T Suzuki, and Y Seta, "Therapeutic effects in a transient middle cerebral artery occlusion rat model by nose-to-brain delivery of antiTNF-alpha siRNA with cell-penetrating peptide-modified polymer micelles," Pharmaceutics, vol. 11, no. 9, 2019.

[5] F. Carvalhal, R. R. Cristelo, D. I. S. P. Resende, M. M. M Pinto, E Sousa, and M Correia-da-Silva, "Antithrombotics from the sea: polysaccharides and beyond," Marine Drugs, vol. 17, no. 3 , 2019.

[6] P. Liu, X. Bai, T. Zhang, L. Zhou, J. Li, and L. Zhang, "The protective effect of Lonicera japonica polysaccharide on mice with depression by inhibiting NLRP3 inflammasome," Annals of Translational Medicine, vol. 7, no. 24, p. 811, 2019.

[7] K. Allegaert, J. De Hoon, A. Debeer, and M. Gewillig, "Renal side effects of non-steroidal anti-inflammatory drugs in neonates," Pharmaceuticals, vol. 3, no. 2, pp. 393-405, 2010.

[8] Y. Ma, L. Li, Z. Niu et al., "Effect of recombinant plasminogen activator timing on thrombolysis in a novel rat embolic stroke model," Pharmacological Research, vol. 107, pp. 291-299, 2016.

[9] J. A. Polderman, V. Farhang-Razi, S. Van Dieren et al., "Adverse side effects of dexamethasone in surgical patients," Cochrane Database of Systematic Reviews, vol. 8, no. 8, Article ID Cd011940, 2018.

[10] H. Ren, G. Shen, J. Tang et al., "Promotion effect of extracts from plastrum testudinis on alendronate against glucocorticoid-induced osteoporosis in rat spine," Scientific Reports, vol. 7, no. 1, p. 10617, 2017.

[11] R.-B. Ding, K. Tian, Y.-W. Cao et al., "Protective effect of Panax notoginseng Saponins on acute ethanol-induced liver injury is associated with ameliorating hepatic lipid accumulation and reducing ethanol-mediated oxidative stress," Journal of Agricultural and Food Chemistry, vol. 63, no. 9, pp. 2413-2422, 2015.

[12] Z. Yang, J. Zhu, H. Zhang, and X. Fan, "Investigating chemical features of Panax notoginseng based on integrating HPLC fingerprinting and determination of multiconstituents by single reference standard," Journal of Ginseng Research, vol. 42, no. 3, pp. 334-342, 2018.

[13] P. F. Yang, X. Y. Song, and N. H. Chen, "[Advances in pharmacological studies of Panax notoginseng saponins on brain ischemia-reperfusion injury]," Yao Xue Xue Bao, vol. 51, no. 7, pp. 1039-1046, 2016.

[14] A. L. Hopkins, "Network pharmacology: the next paradigm in drug discovery," Nature Chemical Biology, vol. 4, no. 11, pp. 682-690, 2008.

[15] W. Y. Lee, C. Y. Lee, Y. S. Kim, and C. E Kim, “The methodological trends of traditional herbal medicine employing network pharmacology," Biomolecules, vol. 9, no. 8, 2019.

[16] D. Moodley, H. Yoshida, S. Mostafavi et al., "Network pharmacology of JAK inhibitors," Proceedings of the National Academy of Sciences, vol. 113, no. 35, pp. 9852-9857, 2016.

[17] X. Niu, J. Zhang, J. Ni et al., "Network pharmacology-based identification of major component of Angelica sinensis and its action mechanism for the treatment of acute myocardial infarction," Bioscience Reports, vol. 38, no. 6, 2018.

[18] S. Zhou, Z. Ai, W. Li et al., "Deciphering the pharmacological mechanisms of taohe-chengqi decoction extract against renal fibrosis through integrating network pharmacology and experimental validation in vitro and in vivo," Frontiers in Pharmacology, vol. 11, p. 425, 2020.

[19] P. Fu, Q. Yuan, Y. Sun et al., "Baicalein ameliorates epilepsy symptoms in a pilocarpine-induced rat model by regulation of IGF1R," Neurochem Res, vol. 45, 2020.

[20] Y. Dong, L. Duan, H. W. Chen et al., "Network pharmacology-based prediction and verification of the targets and mechanism for Panax notoginseng saponins against coronary heart disease," Evidence-Based Complementary and Alternative Medicine, vol. 2019, Article ID 6503752, 13 pages, 2019.

[21] Z. Zhang, L. Zhai, J. Lu et al., "Shen-Hong-Tong-Luo formula attenuates macrophage inflammation and lipid accumulation through the activation of the PPAR- $\gamma /$ LXR- $\alpha /$ ABCA 1 pathway," Oxidative medicine and cellular longevity, vol. 2020, Article ID 3426925, 19 pages, 2020.

[22] Y. Wen, C. Han, T. Liu et al., "Chaiqin chengqi decoction alleviates severity of acute pancreatitis via inhibition of TLR4 and NLRP3 inflammasome: identification of bioactive ingredients via pharmacological sub-network analysis and experimental validation," Phytomedicine, vol. 79, p. 153328, 2020.

[23] J. Ru, P. Li, J. Wang et al., "TCMSP: a database of systems pharmacology for drug discovery from herbal medicines," Journal of Cheminformatics, vol. 6, no. 1, p. 13, 2014.

[24] H.-Y. Xu, Y.-Q. Zhang, Z.-M. Liu et al., "ETCM: an encyclopaedia of traditional Chinese medicine," Nucleic Acids Research, vol. 47, no. D1, pp. D976-d982, 2019.

[25] Z. Liu, F. Guo, Y. Wang et al., "BATMAN-TCM: a bioinformatics analysis Tool for molecular mechANism of traditional Chinese medicine," Scientific Reports, vol. 6, no. 1, p. 21146, 2016. 
[26] X. Wang, Y. Shen, S. Wang et al., "PharmMapper 2017 update: a web server for potential drug target identification with a comprehensive target pharmacophore database," Nucleic Acids Research, vol. 45, pp. W356-w360, 2017.

[27] J. Piñero, J. M. Ramírez-Anguita, J. Saüch-Pitarch et al., “The DisGeNET knowledge platform for disease genomics: 2019 update," Nucleic Acids Research, vol. 48, no. D1, pp. D845-d855, 2020.

[28] D. Szklarczyk, A. L. Gable, D. Lyon et al., "STRING v11: protein-protein association networks with increased coverage, supporting functional discovery in genome-wide experimental datasets," Nucleic Acids Research, vol. 47, no. D1, pp. D607-d613, 2019.

[29] P. Shannon, A. Markiel, O. Ozier et al., "Cytoscape: a software environment for integrated models of biomolecular interaction networks," Genome Research, vol. 13, no. 11, pp. 2498-2504, 2003.

[30] D. W. Huang, B. T. Sherman, and R. A. Lempicki, "Systematic and integrative analysis of large gene lists using DAVID bioinformatics resources," Nature Protocols, vol. 4, no. 1, pp. 44-57, 2009.

[31] O. Trott and A. J. Olson, "AutoDock Vina: improving the speed and accuracy of docking with a new scoring function, efficient optimization, and multithreading," Journal of Computational Chemistry, vol. 31, no. 2, pp. 455-461, 2010.

[32] P. W. Rose, A. Prlić, A. Altunkaya et al., "The RCSB protein data bank: integrative view of protein, gene and 3D structural information," Nucleic Acids Research, vol. 45, no. D1, pp. D271-d281, 2017.

[33] S. Prabhakaran, I. Ruff, and R. A. Bernstein, "Acute stroke intervention," Jama, vol. 313, no. 14, pp. 1451-1462, 2015.

[34] J. L. Saver, "Time is brain-quantified," Stroke, vol. 37, no. 1, pp. 263-266, 2006.

[35] Y. Wang, L. Tu, Y. Li, D. Chen, and S. Wang, "Notoginsenoside R1 protects against neonatal cerebral hypoxic-ischemic injury through estrogen receptor-dependent activation of endoplasmic reticulum stress pathways," Journal of Pharmacology and Experimental Therapeutics, vol. 357, no. 3, pp. 591-605, 2016.

[36] W. Xie, P. Zhou, Y. Sun et al., "Protective effects and target network analysis of ginsenoside Rg1 in cerebral ischemia and reperfusion injury: a comprehensive overview of experimental studies," Cells, vol. 7, no. 12, 2018.

[37] M. Li, Y. Liu, J. Chen et al., "Platelet bio-nanobubbles as microvascular recanalization nanoformulation for acute ischemic stroke lesion theranostics," Theranostics, vol. 8, no. 18, pp. 4870-4883, 2018.

[38] H. Abeysinghe, E. Phillips, H. Chin-Cheng, P. Beart, and C. Roulston, "Modulating astrocyte transition after stroke to promote brain rescue and functional recovery: emerging targets include rho kinase," International Journal of Molecular Sciences, vol. 17, no. 3, p. 288, 2016.

[39] G. Zhang, F. Xia, Y. Zhang et al., "Ginsenoside Rd is efficacious against acute ischemic stroke by suppressing microglial proteasome-mediated inflammation," Molecular Neurobiology, vol. 53, no. 4, pp. 2529-2540, 2016.

[40] Y. F. Chen, K. J. Wu, and W. G. Wood, "Paeonia lactiflora extract attenuating cerebral ischemia and arterial intimal hyperplasia is mediated by paeoniflorin via modulation of VSMC migration and Ras/MEK/ERK signaling pathway," Evidence-Based Complementary and Alternative Medicine, vol. 2013, Article ID 482428, 12 pages, 2013.

[41] Y.-F. Chen, H.-Y. Tsai, K.-J. Wu, L.-R. Siao, and W. G. Wood, "Pipoxolan ameliorates cerebral ischemia via inhibition of neuronal apoptosis and intimal hyperplasia through attenuation of VSMC migration and modulation of matrix metalloproteinase-2/9 and Ras/MEK/ERK signaling pathways," PloS One, vol. 8, no. 9, Article ID e75654, 2013.

[42] M. Bi, A. Gladbach, J. van Eersel et al., "Tau exacerbates excitotoxic brain damage in an animal model of stroke," Nature Communications, vol. 8, no. 1, p. 473, 2017.

[43] Q. Li, Z. Tian, M. Wang et al., "Luteoloside attenuates neuroinflammation in focal cerebral ischemia in rats via regulation of the PPAR $\gamma / \mathrm{Nrf} 2 / \mathrm{NF}-\kappa \mathrm{B}$ signaling pathway," International Immunopharmacology, vol. 66, pp. 309-316, 2019.

[44] K. Zhang, Y. Yang, H. Ge et al., "Artesunate promotes the proliferation of neural stem/progenitor cells and alleviates Ischemia-reperfusion Injury through PI3K/Akt/FOXO-3a/ p27kip1 signaling pathway," Aging, vol. 12, no. 9, pp. 8029-8048, 2020.

[45] E. Bernal-Mizrachi, R. N. Kulkarni, D. K. Scott, F. MauvaisJarvis, A. F. Stewart, and A. Garcia-Ocana, "Human -cell proliferation and intracellular signaling Part 2: still driving in the dark without a road map," Diabetes, vol. 63, no. 3, pp. 819-831, 2014.

[46] S. Wang, S. Lin, M. Zhu et al., "Acupuncture reduces apoptosis of granulosa cells in rats with premature ovarian failure via restoring the PI3K/Akt signaling pathway," International Journal of Molecular Sciences, vol. 20, no. 24, 2019.

[47] J.-i. Takino, T. Sato, K. Nagamine, and T. Hori, "The inhibition of Bax activation-induced apoptosis by RasGRP2 via R-Ras-PI3K-Akt signaling pathway in the endothelial cells," Scientific Reports, vol. 9, no. 1, p. 16717, 2019.

[48] Y. Li, S. Guo, W. Liu et al., "Silencing of SNHG12 enhanced the effectiveness of MSCs in alleviating ischemia/reperfusion injuries via the PI3K/AKT/mTOR signaling pathway," Frontiers in Neuroscience, vol. 13, p. 645, 2019.

[49] M.-R. Lv, B. Li, M.-G. Wang et al., "Activation of the PI3KAkt pathway promotes neuroprotection of the $\delta$-opioid receptor agonist against cerebral ischemia-reperfusion injury in rat models," Biomedicine \& Pharmacotherapy, vol. 93, pp. 230-237, 2017.

[50] C. Feng, H. Wan, Y. Zhang et al., "Neuroprotective effect of Danhong injection on cerebral ischemia-reperfusion injury in rats by activation of the PI3K-Akt pathway," Frontiers in Pharmacology, vol. 11, p. 298, 2020.

[51] M.-M. Wang, M. Zhang, Y.-S. Feng et al., "Electroacupuncture inhibits neuronal autophagy and apoptosis via the PI3K/AKT pathway following ischemic stroke," Frontiers in Cellular Neuroscience, vol. 14, p. 134, 2020.

[52] Q. Wu, Z. Mao, J. Liu, J. Huang, and N. Wang, "Ligustilide attenuates ischemia reperfusion-induced hippocampal neuronal apoptosis via activating the PI3K/Akt pathway," Frontiers in Pharmacology, vol. 11, p. 979, 2020.

[53] W. Zhang, Y. Wu, H. Chen et al., "Neuroprotective effects of SOX5 against ischemic stroke by regulating VEGF/PI3K/AKT pathway," Gene, vol. 767, Article ID 145148, 2020.

[54] S. Hu, Y. Wu, B. Zhao et al., "Panax notoginseng saponins protect cerebral microvascular endothelial cells against oxygen-glucose deprivation/reperfusion-induced barrier dysfunction via activation of PI3K/Akt/Nrf2 antioxidant signaling pathway," Molecules, vol. 23, no. 11, 2018.

[55] F. Tan, W. Fu, N. Cheng, D. Meng, and Y. Gu, "Ligustrazine reduces blood-brain barrier permeability in a rat model of focal cerebral ischemia and reperfusion," Experimental and Therapeutic Medicine, vol. 9, no. 5, pp. 1757-1762, 2015. 
[56] Y.-T. Yan, S.-D. Li, C. Li et al., "Panax notoginsenoside saponins Rb1 regulates the expressions of $\mathrm{Akt} / \mathrm{mTOR} / \mathrm{PTEN}$ signals in the hippocampus after focal cerebral ischemia in rats," Behavioural Brain Research, vol. 345, pp. 83-92, 2018.

[57] T. Wu, Z. Jia, S. Dong et al., "Panax notoginseng saponins ameliorate leukocyte adherence and cerebrovascular endothelial barrier breakdown upon ischemia-reperfusion in mice," Journal of Vascular Research, vol. 56, no. 1, pp. 1-10, 2019.

[58] Y. Zhang, K. Sun, Y.-Y. Liu et al., "Ginsenoside Rb1 ameliorates lipopolysaccharide-induced albumin leakage from rat mesenteric venules by intervening in both trans- and paracellular pathway," American Journal of Physiology - Gastrointestinal and Liver Physiology, vol. 306, no. 4, pp. G289-G300, 2014.

[59] T. Yoshida and P. Delafontaine, "Mechanisms of IGF-1mediated regulation of skeletal muscle hypertrophy and atrophy," Cells, vol. 9, no. 9, 2020.

[60] L. He, X. Liu, J. Yang et al., "Imbalance of the reciprocally inhibitory loop between the ubiquitin-specific protease USP43 and EGFR/PI3K/AKT drives breast carcinogenesis," Cell Research, vol. 28, no. 9, pp. 934-951, 2018.

[61] P. Wee and Z. Wang, "Epidermal growth factor receptor cell proliferation signaling pathways," Cancers, vol. 9, no. 5, 2017.

[62] J. Wang, H. Du, Y. Nie et al., "Mitochondria and MAPK cascades modulate endosulfan-induced germline apoptosis in Caenorhabditis elegans," Toxicology Research, vol. 6, no. 4, pp. 412-419, 2017.

[63] X. Xiao, W. Wang, Y. Li et al., "HSP90AA1-mediated autophagy promotes drug resistance in osteosarcoma," Journal of Experimental \& Clinical Cancer Research, vol. 37, no. 1, p. 201, 2018.

[64] L. Shu, D. Wang, N. F. Saba, and Z. G. Chen, "A historic perspective and overview of $\mathrm{H}$-Ras structure, oncogenicity, and targeting," Molecular Cancer Therapeutics, vol. 19, no. 4, pp. 999-1007, 2020. 